\title{
A docked mutation phenocopies dumpy oblique alleles via altered vesicle trafficking
}

\author{
Suresh Kandasamy ${ }^{1}$, Kiley Couto ${ }^{1}$, Justin Thackeray ${ }^{\text {Corresp. } 1}$ \\ ${ }^{1}$ Department of Biology, Clark University, Worcester, Massachusetts, United States \\ Corresponding Author: Justin Thackeray \\ Email address: jthackeray@clarku.edu
}

The Drosophila extracellular matrix protein Dumpy (Dpy) is one of the largest proteins encoded by any animal. One class of $d p y$ mutations produces a characteristic shortening of the wing blade known as oblique $\left(d p y^{\circ}\right)$, due to altered tension in the developing wing. We describe here the characterization ofdocked $(d o c)$, a gene originally named because of an allele producing a truncated wing. We show thatdoc corresponds to the gene modelCG5484, which encodes a homolog of the yeast protein Yif1 and plays a key role in ER to Golgi vesicle transport. Genetic analysis is consistent with a similar role for Doc in vesicle trafficking:dockedalleles interact not only with genes encoding the COPII core proteins sec23 and sec13, but also with the SNARE proteins synaptobrevin and syntaxin.

Further, we demonstrate that the strong similarity between thedoc ${ }^{1}$ and $d p y^{0}$ wing phenotypes reflects a functional connection between the two genes; we found that variousdpy alleles are sensitive to changes in dosage of genes encoding other vesicle transport components such assec13andsar1. Doc's effects on trafficking are not limited to Dpy; for example, reduceddoc dosage disturbed Notch pathway signaling during wing blade and vein development. These results suggest a model in which the oblique wing phenotype indoc ${ }^{1}$ results from reduced transport of wild-type Dumpy protein; by extension, an additional implication is that thedpy alleles can themselves be explained as hypomorphs. 
1 A docked mutation phenocopies dumpy oblique alleles via 2 altered vesicle trafficking

3

4 Suresh K. Kandasamy ${ }^{1}$, Kiley Couto ${ }^{1}$ and Justin R. Thackeray ${ }^{1}$

5

$6 \quad{ }^{1}$ Department of Biology, Clark University, Worcester, Massachusetts, USA

7

8 


\section{Abstract}

11 The Drosophila extracellular matrix protein Dumpy (Dpy) is one of the largest proteins encoded

12 by any animal. One class of $d p y$ mutations produces a characteristic shortening of the wing blade

13 known as oblique $\left(d p y^{o}\right)$, due to altered tension in the developing wing. We describe here the

14 characterization of docked (doc), a gene originally named because of an allele producing a

15 truncated wing. We show that doc corresponds to the gene model CG5484, which encodes a

16 homolog of the yeast protein Yifl and plays a key role in ER to Golgi vesicle transport. Genetic

17 analysis is consistent with a similar role for Doc in vesicle trafficking: docked alleles interact not

18 only with genes encoding the COPII core proteins sec23 and sec13, but also with the SNARE

19 proteins synaptobrevin and syntaxin. Further, we demonstrate that the strong similarity between

20 the $d o c^{l}$ and $d p y^{o}$ wing phenotypes reflects a functional connection between the two genes; we

21 found that various $d p y$ alleles are sensitive to changes in dosage of genes encoding other vesicle

22 transport components such as sec 13 and sarl. Doc's effects on trafficking are not limited to Dpy;

23 for example, reduced doc dosage disturbed Notch pathway signaling during wing blade and vein

24 development. These results suggest a model in which the oblique wing phenotype in $d o c^{l}$ results

25 from reduced transport of wild-type Dumpy protein; by extension, an additional implication is

26 that the $d p y^{o}$ alleles can themselves be explained as hypomorphs. 
28 Introduction

29

30

31

32

33

34

About one third of all eukaryotic proteins pass through the endoplasmic reticulum (ER) on their way to a variety of ultimate destinations. Anterograde traffic from the ER to the Golgi apparatus is highly conserved across all eukaryotes and depends on coat protein complex II (COPII) vesicles, while retrograde transport from the Golgi back to the ER relies on COPIcoated vesicles (Lord et al. 2013; Venditti et al. 2014). COPII vesicles are assembled in a stepwise manner, beginning with activation of the small G-protein Sar1. This leads to recruitment of a Sec23/Sec24 heterodimer, followed by the assembly of an outer coat heterotetramer constructed from Sec13/Sec31 molecules. However, it is clear that this description is incomplete for some cargoes; for example, traffic of collagen requires additional factors (TANGO1 and cTAGE5) to interact with Sec23/24, presumably to allow such a large protein to be packaged inside the vesicle (Malhotra \& Erlmann 2011). Upon reaching the Golgi the vesicle is tethered by a combination of a multi-protein complex known as TRAPP, a Rab family member called Ypt and several other Ypt-interacting proteins. Finally, fusion of the vesicle to its target membrane depends on a set of proteins known as SNAREs (soluble N-ethylmaleimide-sensitive factor attachment protein receptor) (Lord et al. 2013).

A particularly challenging cargo in Drosophila is presented by Dumpy (Dpy), with some isoforms being 23,000 amino acids long and estimated at 2.5 MD (Wilkin et al. 2000). Dpy is probably the largest protein made by Drosophila and is among the largest proteins made by any organism; it was recently shown that Dpy is trafficked via a bulky cargo-specific pathway that depends on the Drosophila Tango homolog, Tangol (Rios-Barrera et al. 2017). Dpy is membrane-anchored but the vast majority of the molecule projects into the extracellular matrix (ECM), where it plays a role in attachment between epithelial cells and adjacent tissues (Wilkin et al. 2000). Loss of $d p y$ function is lethal, but its function can be observed from loss of function restricted to the wing, which results in blisters due to separation of the apical ECM (aECM) from the epidermis (Bokel et al. 2005; Prout et al. 1997). Much of the Dpy protein consists of many copies of three repeats; in addition to over 300 EGF (Epidermal Growth Factor) repeats there are two distinct repeats unique to Dumpy - 185 copies of a Dumpy-specific motif referred to as DPY, and about 40 copies of a sequence known as PIGSFEAST (Wilkin et al. 2000). Some of the EGF repeats are substrates for Eogt, an extracellular-specific O-Glc-NAc (O-linked N- 
59

60

61

62

63

64

65

66

67

68

69

70

80

(8)

acetylglucosamine) transferase, and this modification affects Dpy function in the aECM (Sakaidani et al. 2011). dumpy (dpy) has been the subject of intensive genetic study over many decades, due to the combination of several easily identified mutant phenotypes and the huge mutational target of the gene itself. The locus is genetically complex, with alleles falling into three main classes: oblique $\left(d p y^{o}\right)$, vortex $\left(d p y^{v}\right)$ and lethal $\left(d p y^{l}\right)$, and many showing more than one of the three phenotypes (Carlson 1959). The oblique class gave the gene its name, producing a short and distally clipped wing, with a reduced number of cells in the wing and an indentation close to the margin between veins L3 and L4 (Waddington 1940); this is due to abnormal tension between cells within the developing wing, which skews its final shape (Etournay et al. 2015).

The Drosophila wing is a large and completely dispensable organ, with a highly stereotyped pattern of veins. In combination with an array of genetic tools - particularly a collection of wing-specific GAL4 drivers - these features have allowed studies of the fly wing to greatly expand our understanding of organogenesis and patterning (Baker 2007; Neto-Silva et al. 2009). The Drosophila wing develops from a group of about 20 cells in the embryo into an imaginal disc of about 75,000 cells by the end of the third larval instar. During pupariation the wing disc everts and cuticle is secreted to form the dorsal and ventral surfaces, which are held together by a meshwork of ECM molecules. The vesicular trafficking machinery plays a central role at all stages in this process, being necessary for not only for placement of membrane-bound receptors such as Notch, Frizzled, Patched, Thickveins, Punt and EGFR, but also for secretion of various morphogens including Wingless (Wg), Hedgehog (Hh), Decapentaplegic (Dpp) and Vein (Vn). lethal complementation group distinct from amon was also identified in an adjacent genomic region. Among the mutations recovered was a single viable allele, producing an indentation in the distal part of the wing; the new gene was named docked because of this phenotype (Rayburn et al. 2003). The lethal alleles of $d o c\left(d o c^{13}, d o c^{16}\right.$ and $\left.d o c^{99}\right)$ fail to complement each other, but are viable when heterozygous with the viable allele $d o c^{l}$, although they all fail to complement the wing phenotype of $d o c^{1}$ (Rayburn et al. 2003). Noting the similarity between the $d o c^{l}$ and $d p y^{o}$ wing phenotype we began investigating the $d o c$ alleles, to reveal whether Doc may 
89 participate with Dpy during wing development. We describe here our discovery that doc encodes

90 the Drosophila homolog of yeast YIF1 (Yip1-interacting factor), and this finding led us to the

91 discovery that the dumpy oblique phenotype can be explained by aberrant trafficking of wild-

92 type Dpy.

93

94

95 Materials and Methods

96

97

Fly stocks

98

Flies were raised on a standard cornmeal, molasses, yeast and agar medium at $25^{\circ} \mathrm{C}$.

100

$d o c^{1} / T M 3 S b, d o c^{16} / T M 3 S b$, Ser and doc $99 / T M 6 T b$ were kind gifts from Dr. Michael Bender

101

(Univ. of Georgia). A strain carrying the $U A S-d N S F 2^{E / Q}$ construct (Stewart et al. 2001) was

102

kindly provided by Hugo Bellen (Baylor College of Medicine, Texas). A UAS-RNAi-doc strain

103

(ID\#2679) was obtained from the Vienna Drosophila Research Center. $d p y^{D v l G 5}$ was isolated

104

from an EMS mutagenesis screen (T. Abbeyquaye and J. Thackeray, unpublished). All other

105 stocks were obtained from the Bloomington Drosophila Stock Center at Indiana University.

\section{Generating a wild type $C G 5484$ transgenic rescue construct}

DNA was extracted from wild-type canton S strain flies and PCR was carried out using

Native Pfu polymerase (Stratagene Inc, La Jolla CA) according to the manufacturer's instructions. The CG5484 transcription unit and about $1 \mathrm{~kb}$ of intergenic sequence on either side were amplified in two overlapping PCR products and joined using a common NsiI cut site to generate a full-length wild-type CG5484 rescue construct. The forward and reverse primers for

113 the more 5' product were 5'TTCGCGCTGGCTCAAACTGCCTA3' and 5'-

114 CCAAGAGCTCCCAGTGCAACCA-3' respectively; the underlined base is a mismatch used to

115 incorporate a SacI site into the product. The 3' half of CG5484 gene was amplified the primer

116 pair 5'GCGAAGTAGTACTTAAGCTTGGC3' and 5'-GGATGGATTCATCTAGACCTCGG-

117 3' respectively; the underlined base in the former is a mismatch used to incorporate a HindIII

118 site, in the latter primer the underlined base introduces an $X b a \mathrm{I}$ restriction site. A $4.1 \mathrm{~kb}$ 
$119 S a c I I / X b a I$ fragment that begins 1124bp 5' of the predicted translation start and ends 1352bp 3'

120 of the predicted stop codon was ligated into the pCaSpeR4 germline transformation vector. The

121 genomic insert in this vector was verified by sequencing and then injected into white 1118 embryos

122 to produce transgenic lines expressing wild-type CG5484; transgenic injection was performed by

123 Genetic Services Inc, Sudbury, MA.

124

125

126 Results

127

128 Alleles of $d o c$ interact genetically with dumpy mutations

We noticed a strong similarity in wing phenotype between $d o c^{l}$ homozygotes and the

130 oblique class of dumpy (dpy) mutants, which suggested to us that Doc and Dpy may be involved

131 in a common pathway during wing development. We outcrossed four $d o c$ alleles and confirmed

132 their allelic status, the lethality of $d o c^{13,16,99}$ and the oblique wing phenotype of $d o c^{l}$ (Fig. 1).

133 These initial experiments also revealed that $d o c^{1}$ is semi-dominant: $d o c^{1 /+}$ heterozygotes show a

134 very slight indentation in the distal part of the wing. Next we looked for genetic interaction

135 between $d o c$ and $d p y$. We found that the oblique wing phenotype of two dominant $d p y$ alleles,

$136 d p y^{D}$ and $d p y^{D v l G 5}$ as well as a recessive allele $d p y^{o l v r}$ is strongly enhanced when placed in a

137 background heterozygous for $d o c^{l}$, producing a significant further reduction in wing length (Fig.

138 1). This interaction is consistent with Doc and Dpy playing roles in a common signaling

139 pathway. However, two lethal alleles of $d o c, d o c^{16}$ and $d o c^{99}$, did not interact with any of these

$140 d p y$ alleles (data not shown), suggesting that the $d o c^{1}$ mutation is qualitatively different from the

141 other two doc alleles.

142

143 Doc is encoded by the gene model CG5484

144

The $d o c$ alleles were identified originally by either their lethality $\left(d o c^{13,16,19}\right)$ or their wing

146 phenotype $\left(d o c^{l}\right)$ when heterozygous with two overlapping third chromosome deficiency 
147 chromosomes, $D f(3 R)$ ro $80 b$ and $D f(3 R) T l-X$ (Rayburn et al. 2003), implying that the $d o c$

148 transcription unit lies within the genomic interval common to both deficiencies. We amplified

149 genomic DNA from the doc mutant chromosomes, using primers in coding regions from several

150 of the approximately 20 genes within this interval. We targeted loci predicted to encode proteins

151 that seemed most likely to play a role in regulating signaling pathways in wing development:

152 CG6420, CG5484, beat VII and scribbled. Nucleotide sequences from these candidate genes in

153 various $d o c^{1}, d o c^{16} / T M 3 S b$ and $d o c^{99} / T M 6 S b$ backgrounds were compared to wild-type; we did

154 not observe unique mutations in any of the candidate genes except one: CG5484. The doc ${ }^{16}$

155 chromosome carries a nonsense mutation in the predicted CG5484 open reading frame, affecting

156 amino acid position 162 and therefore eliminating more than half of the predicted full length

157 protein (Fig. 2A). However, we were unable to identify mutations in CG5484 in either the $d o c^{l}$

158 or $d o c^{99}$ chromosomes. To establish whether CG5484 corresponds to $d o c$, we generated a wild

159 type genomic construct containing all predicted CG5484 exons and introns, as well as

160 approximately $1 \mathrm{~kb}$ of additional 5 ' and 3' sequence beyond the predicted transcription unit. A

161 single copy of this transgene completely rescued both the oblique wing phenotype of $d o c^{l}$

162 homozygotes and the lethality of $d o c^{16}$ (Fig. 2B,C,D). The transgenic construct did not rescue the

163 lethality of $d o c^{99}$, but this is likely due to a tightly linked lethal mutation in another locus,

164 because the $C G 5484$ construct was able to rescue the lethality of $d o c^{16} / d o c^{99}$ transheterozygotes

165 (data not shown). Taken together with the nonsense mutation in the $d o c^{16}$ chromosome, these

166 germline rescue experiments demonstrate that CG5484 encodes Doc. Henceforth we refer to

167 CG5484 as docked.

168

169 doc encodes a Yif1 homolog

170

171

172

173

174

175

176

177

The FlyBase annotation of $d o c$ shows that the gene produces three slightly different mRNAs via alternative splice site usage at the 3' end of exon 1 (McQuilton et al. 2011), predicting a set of three very similar Doc isoforms: A (397aa), B (393aa) and C (402aa). Genbank searches using the longest isoform revealed that Doc has no other homologs in Drosophila (not shown). However, these searches also clearly demonstrated that Doc is a member of the YIP (Ypt-interacting protein) superfamily that was first identified in the budding yeast, $S$. cerevisiae (Matern et al. 2000). Comparison of Doc with the most similar YIP family 
178 members from yeast (YIF1) and human (Yif1A) shows relatively poor conservation over the N-

179 terminal third of Doc, but the region homologous to the more C-terminal two thirds shows

180 substantial conservation among all three species (Fig. S1). A characteristic feature of YIP family

181 members is the presence of five likely membrane-spanning domains; a hydropathy plot of the

182 Doc sequence reveals that these are conserved within Doc (Fig. S2). Doc shows greatest

183 similarity to yeast Yif1 (Yip1 interacting factor), which is an integral membrane protein required

184 for membrane fusion of ER- derived vesicles (Barrowmann et al. 2003) and is essential for ER to

185 Golgi vesicle-mediated transport in S. cerevisiae (Heidtman et al. 2005). While we were

186 preparing this manuscript others reported the same finding, that CG5484 is a Yif1 homolog in

187 Drosophila (Wang et al., 2018).

188

189

We used PCR amplification from mRNA to confirm that the A, B and C splice forms are generated in wild-type adult tissue; these experiments also suggest that the B form is the most abundant of the three (Fig. S3). There are no reports of alternative splicing in the exons encoding the N-terminal end of the yeast or human YIF1 homologs; so we examined other insect species to determine whether the three alternative splice forms are evolutionarily conserved. All eight Drosophila species we examined show conservation of the three alternative splice donor (GT) sequences at identical locations in their respective doc homologs, suggesting that the three Doc isoforms are conserved in the other species and therefore likely to have distinct functional roles

197 (Fig S3). In summary, the Doc sequence strongly suggests it is a YIF1 homolog and is therefore very likely to be involved in vesicle trafficking in Drosophila.

\section{Interaction of $d o c$ with genes encoding COPII core proteins}

S. cerevisiae yifl mutants exhibit defective COPII vesicle transport (Matern et al. 2000) and its Yif1 protein product has been described as either a COPII vesicle component, or a resident of the Golgi necessary for ER-derived COPII vesicle docking (Heidtman et al. 2003; Jin et al. 2005; Matern et al. 2000). To determine whether Doc plays a similar role in Drosophila we looked for genetic interaction between $d o c$ and the genes encoding some of the core components of COPII vesicles. The COPII coat assembles by a stepwise deposition of Sar1-GTP, Sec23Sec24, and Sec13-Sec31 onto ER exit sites (Gurkan et al. 2006). Sec23-Sec24 and Sec13-Sec31 
209 are heteromeric protein complexes that are major constituents of the inner and outer layers of the

210 COPII coat (Matsuoka et al. 1998). We found a strong interaction between doc and sec23

211 mutations: there is a noticeable enhancement of the oblique wing phenotype in $\operatorname{doc}^{1} \sec 23^{j 13 C 8}$

212 double heterozygotes (Fig. 3), whereas the double heterozygous combination of $d o c^{l}$ and $\sec 23^{9 G}$

213 alleles was lethal. Similarly, in a background heterozygous for sec $13^{01031}$ the oblique wing

214 phenotype of $d o c^{l}$ heterozygotes was markedly more severe compared to $d o c^{l} /+$ alone (Fig. 3).

215 In the case of sarl we observed no change to the severity of the wing in $\operatorname{doc}^{1} / \operatorname{sarl}^{05712}$ flies, but

216 this combination showed some evidence of semi-lethality: the number of $\operatorname{doc}^{1}+/+\operatorname{sarl}^{05712}$

217 double heterozygotes was reduced by about $40 \%$ compared to the combined number of

$218 d^{1} c^{1} / T M 3$ and $\operatorname{sarl}^{05712 / T M 3}$ siblings (6 vs 30 respectively). These synergistic interactions

219 strongly suggest a role for Doc in COPII vesicle trafficking. We did not observe any interaction

220 in animals that were doubly heterozygous for either $d o c^{16}$ or $d o c^{99}$ and also $\sec 23^{j 13 C 8}$, sec1 $13^{01031}$

221 or $\operatorname{sar1} 1^{05712}$.

222

223

\section{Doc has roles in vesicle fusion}

224

225

Studies from yeast have revealed that Yif1 forms a complex with Yip1 and interacts

226 with Rab GTPases in the acceptor Golgi membrane, suggesting a role for Yifl in tethering of COPII vesicles (Matern et al. 2000). Accurate matching of vesicles to the correct target depends on $\mathrm{v}$-SNARE proteins in the COPII membrane forming a complex with specific t-SNAREs in the target membrane. We therefore generated heterozygous combinations of doc mutations with mutations of genes encoding either the v-SNARE component Synaptobrevin $(s y b)$, or one of the t-SNARE proteins Syntaxin $(S y x)$, using the syntaxin 1 homolog encoded by $\operatorname{Syx} 1 \mathrm{~A}$. We observed clear interaction between $d o c^{1}$ and $S y x 1 A$, first, by a modest enhancement of the $d o c^{l}$ oblique wing phenotype in $d o c^{1}+/+S y x 1 A^{4229}$ flies (Fig. 3G,H,I) and second, because the $d o c^{99}$ mutation showed semi-lethality even in heterozygous condition if the dosage of Syx1A was also reduced using the null allele $\operatorname{Sy} \times 1 A^{4229}$ : the cross produced only $36 \%$ of the number of $d o c^{99}+/+$ Syx $1 A^{4229}$ adults compared to their $d o c^{99} / T M 3$ sibs (five $d o c^{99}+/+S y x 1 A^{4229}$ adults compared to $28 S_{y x} 1 A^{4229} / T M 3$ or $\left.d o c^{99} / T M 3\right)$. By contrast, we found no interaction between any doc allele tested and $s y b$ (data not shown). These results are consistent with a role for Doc in COPII vesicle 
239 fusion with the ER, and suggest a more critical involvement with events occurring on the target 240 membrane.

241

242

243 membrane fusion. One aspect of this is the need for cis-residing protein complexes to be broken apart to be recycled for future trans-complex formation, maintaining anterograde vesicle transport. NSF is an ATPase involved in dissociation of SNAREs (Banerjee et al. 1996) and thus NSF may permit recycling of SNAREs to maintain a steady rate of protein transport. We perturbed dNSF function in the wing margin by expressing the dominant negative $U A S$ $d N S F 2^{E / Q}$ construct (Stewart et al. 2001) under the control of $c 96-G A L 4$, which drives expression in the wing margin (Gustafson \& Boulianne 1996), generating nicks. We found that this wing nick phenotype is enhanced in backgrounds that are heterozygous for any of $d o c^{1}, d o c^{16}$ or $d o c^{99}$ (Fig. 3J-K). The interaction of $d o c$ with $d N S F 2$ is consistent with a role for Doc in vesicle trafficking, and suggests a role for Doc that matches that of yeast Yifl in vesicle fusion

253 (Barrowmann et al. 2003).

254

\section{doc is required broadly during development}

Flies homozygous for either the $d o c^{16}$ or $d o c^{99}$ alleles, as well as $d o c^{16} / d o c^{99}$ transheterozygotes, do not survive development, with a lethal phase during embryogenesis (data not shown). Similarly, we found that expression of a $U A S-d o c-R N A i$ construct using the broadly expressed $w g-G A L 4$ driver is lethal (data not shown). Furthermore, restricting the $d o c$ knockdown to trachea using the breathless-GAL4 driver (Matusek et al. 2006; Reichman-Fried \& Shilo 1995) was also lethal, consistent with a general requirement for Doc during organogenesis. The adult wing provides a convenient way to examine cell-lethal functions in the adult, because it is completely dispensable. RNAi knockdown of $d o c$ in the wing using the dorsal compartment driver MS1096-GAL4 resulted in complete loss of wing structures (not shown). However, restricting knockdown of $d o c$ to the dorsal-ventral (DV) wing boundary using the C96-GAL4 driver (Gustafson \& Boulianne 1996) resulted in partial loss only of marginal tissue (Fig. 4).

\section{A role for Doc in Notch ligand transport}



a role for Doc during export of developmentally important signaling ligands in the wing. Spatially restricted expression of the Notch (N) receptor and its ligands, Serrate (Ser) and Delta (D) play key roles in wing margin development (Artavanis-Tsakonas et al. 1999; de Celis et al. 1996; Neumann \& Cohen 1996; Panin \& Irvine 1998) and marginal wing nicks very similar to those we observed with $C 96>d o c-R N A i$ are observed in some alleles of both $N$ and $\operatorname{Ser}$ (Thomas et al. 1991; van de Hoef et al. 2013). We therefore examined whether there are genetic interactions between the genes encoding these molecules and docked.

Wings of $\mathrm{Dl}^{7}$ mutants have thickened veins and delta-like wing venation patterns and we found that this $D l$ venation phenotype is enhanced by all three $d o c$ alleles tested: $d o c^{1}, d o c^{16}$ and $d c^{99}$ (Fig. 4A-C). This was manifested by much increased thickening of vein L2, as well as frequent thickening and/or delta formation at the distal ends of veins L3 and L4. A similar enhancement was observed when doc dosage was reduced in a background that was heterozygous for both $D l^{R e v F 10}$ and $\operatorname{Ser}^{R X 82}$ (Fig. 4D-F). We found a particularly strong interaction between $d o c$ and $N$. Halving the dosage of docked with either $d o c^{1}$ or $d o c^{16}$ was lethal in a $N^{n d-3}$ heterozygous background, while in a $d o c^{99}$ heterozygous background only an occasional $N^{n d-3} /+; d o c^{99} /+$ escaper was recovered; these escapers did not have any observable change in wing phenotype (not shown). These results are consistent with Notch signaling being reliant on Doc-dependent vesicle trafficking.

Our finding that at least some Notch signaling depends on Doc suggests a possible cause of the wing nicks observed in the $C 96>R N A i-d o c$ animals: failed trafficking of $\mathrm{N}$ or its ligands. We tested this model directly, by overexpressing Dl or Ser, using $U A S-D l$ or $U A S-S e r$ constructs driven by the C96-GAL4 driver, in a background also expressing the UAS-doc-RNAi construct. We found that overexpression of Delta was sufficient to completely rescue the wing nicks resulting from RNAi knockdown of $d o c$, whereas in the case of Serrate the rescue was strong, but not complete (Fig. 4G-I). By contrast, overexpression of Notch using the same method did not rescue the $d o c$-RNAi wing margin defect, although some degree of rescue was apparent in most animals (Fig. 4J). These findings strongly suggest that the wing nicks observed 
301 in $C 96>R N A i-d o c$ animals are due to reduced availability of Notch ligands, presumably as a

302 result of lowered vesicle trafficking.

303

304 The interaction between $d o c$ and $d p y$ is related to transport of Dpy

305

306

307

308

309

310

311

312

313

314

315

316

317

318

319

320

321

322

323

324

325

326

327

328

329

330

Animals homozygous for $d o c^{1}$ are a remarkably accurate phenocopy of the oblique class of $d p y$ mutations, and we have shown in this study that a strong genetic interaction exists between the two genes (Fig. 1). Dpy resides in the extracellular matrix (Wilkin et al. 2000), so it can be assumed to pass through the vesicular trafficking pathway to reach its final destination. Given the similarity in their phenotypes, an obvious question is whether the $d o c^{l}$ wing phenotype arises from reduced transport of Dpy. If this is true, then we might expect other mutations that disturb vesicle trafficking to worsen the severity of the oblique wing phenotype in dumpy mutants. We find that this is indeed the case: whereas $d p y^{D}$ heterozygotes show a barely detectable oblique phenotype, transheterozygous $d p y^{D /+}$; $\sec 13^{01031 /+}$ and $d p y^{D /+}$; $\operatorname{sar} 1^{05712 /+}$ animals both show a much more substantial oblique phenotype (Fig. 5). By contrast, no observable change in the severity of the oblique wing phenotype was observed in transheterozygous combinations of $d p y^{D}$ with either of two sec 23 alleles, $\sec 23^{9 G}$ or $\sec 23^{j 13 C 8}$ (data not shown). The interaction between $d p y^{D}$ and both $\sec 13^{01031}$ and $\operatorname{sar} 1^{05712}$ is consistent with a model in which the $d o c^{l}$ oblique wing phenotype is due to an effect occurring via Dumpy.

The interaction of $d p y^{D}$ with alleles of $d o c$, sec13 and sarl all suggest a particular sensitivity of Dpy to reduced COPII vesicle transport, but we wondered if the same effect might be seen if vesicle trafficking at other stages was reduced. Jagunal (Jagn) is a conserved ER membrane protein required for replenishment of the ER membrane (Lee \& Cooley 2007), so we tested whether jagn mutants show any interaction with $d p y^{D}$. Although there was no observable wing phenotype in adults (not shown), we found that the transheterozygous combination of

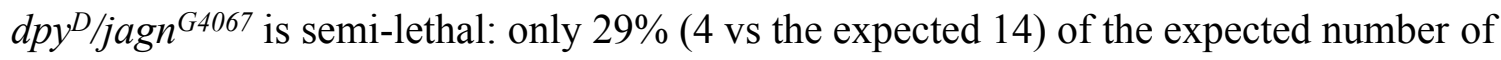
$d p y^{D / j a g n^{G 4067}}$ adults emerged compared to their $d p y^{D /+}$ or $j a g n^{G 4067 /+}$ siblings. This result buttresses the idea that Dpy is highly sensitive to reduced COPII-dependent traffic, because Jagn is needed to maintain future rounds of COPII transport; it also shows that this effect is not specific to a particular stage of extracellular transport.

Peer) reviewing PDF | (2018:10:31761:1:1:NEW 19 Aug 2021) 
Halving the dosage of genes encoding components of the vesicle trafficking machinery

332

333

334

335

336

337

338

339

340

341

342

343

344

345

346

347

348

349

350

351

352

353

354

355

356

357

358

359

360 would be expected to affect any protein that passes through the ER, and not just Dpy. We therefore looked for genetic interaction between $d o c$ and other genes encoding proteins that are similar to Dpy in any of size, amino acid sequence or extracellular location. Dpy is one of a small group of proteins that contain a ZP (zona pellucida) domain, a motif that is unique to a subset of extracellular proteins (Jovine et al. 2002); all ZP domain-containing proteins can therefore be assumed to be trafficked between the ER and Golgi to reach their final destination. Sixteen Drosophila genes encoding proteins with a ZP domain are identifiable in release 6 of the Drosophila genome and we examined P-insertion alleles of ten for genetic interaction with doc. Nine of these ten - dusky-like, Dusky, piopio, cypher, trynity, nyobe, nyeo, nomp-A and $C G 17111$ - showed no interaction with any of $d o c^{1}, d o c^{16}$ or $d o c^{99}$ when in various transheterozygous combinations (data not shown). However one member of this group, miniature, did show significant interaction with doc. Miniature is a transmembrane protein required for proper assembly of the chitin-based cuticle; adults homozygous or hemizygous for viable miniature $(m)$ alleles have short wings (Slatis \& Willermet 1954). Wings of hemizygous $m^{1}$ males that were also heterozygous for either $d o c^{1}$ or $d o c^{16}$ had much more abnormal wings than $m^{1} / Y$ alone, being both narrow and misshapen (Fig. 5F-H). Although the reduced size of the wings of $m^{1} / Y$; $d o c^{99} /+$ males was typical of $m^{1}$ hemizygotes, there was a noticeable increase in the amount of darkly-pigmented tissue that is characteristic of some $m$ alleles (Fig. 5I). In addition, two transheterozygous combinations of $d o c$ and $m$ were lethal: $m^{259-4 /+} ; d o c^{16 /+}$ and $m^{259-4} /+$; $d o c^{99} /+$. By contrast, we found no interaction between $d o c^{1}$ and $m^{259-4}$ (data not shown).

Finally, because Dpy is an exceptionally large protein known to use a distinct trafficking pathway for bulky cargoes (Rios-Barrera et al. 2017) we wondered whether the interaction between $d o c^{l}$ and $d p y$ might also be related to the exceptional size of Dumpy. We were able to identify in Flybase only one other secreted Drosophila protein approaching its size: Mucin14A, which has a largest isoform of 16,233aa (Syed et al. 2008). We found no change in the phenotype of any of the three doc alleles when they were placed in a background heterozygous for a viable allele of Mucl $4 A^{f 00286}$, suggesting that the interaction of Doc with Dumpy is not solely due to its large size (not shown). Because the size of Dpy could conceivably be causing other Doc-dependent effects distinct from secretion, we also tested for interactions with genes 
362 encoding six relatively large intracellular proteins sallimus (longest predicted isoform given in

363 Flybase (St Pierre et al. 2014) 18,488aa), ankyrin2 (13,559aa), muscle-specific protein 300

364 (13,540aa), stretchin (9,839aa), bent (8,933aa) and shortstop (5,501aa), but found no interaction

365 when in transheterozygous combinations with various $d o c$ alleles (not shown). These findings

366 suggest that the interaction between $d o c^{1}$ and $d p y$ is unlikely to be due to the size of Dpy or the

367 presence of a ZP-domain per se.

368 


\section{Discussion}

370

371

372

373

374

375

376

377

378

379

380

381

382

383

384

385

386

387

388

389

390

391

392

393

394

395

396

397

398

399

We have shown here, by identification of a nonsense mutation in $d o c^{16}$ and germline rescue of the mutant phenotypes in three independent $d o c$ alleles, that the gene model CG5484 corresponds to docked. The predicted Doc amino acid sequence shows strong sequence similarity to yeast Yif1; it is also of similar size and contains the five predicted transmembrane domains that are typical of Yif1 homologs. Yeast Yif1 is necessary for COPII vesicle transport (Matern et al. 2000) and forms a complex with a paralogous protein, Yip1; this integral membrane complex is thought to be required either for formation of COPII vesicles at ER exit sites (Heidtman et al. 2003) or for fusion of those vesicles with the Golgi (Barrowmann et al. 2003). Our genetic data are clearly consistent with a role for Doc in COPII trafficking; for example, the interaction between doc alleles and mutants encoding the COPII vesicle components Sar1 and Sec13. Our results are also consistent with a role for Doc/Yif1 in vesicle fusion, given that we did not see an interaction with mutations of the v-SNARE-encoding gene $S y b$, whereas moderate enhancement of the $d o c^{l}$ wing phenotype and semi-lethality with $d o c^{99}$ occurred with hypomorphic alleles of the t-SNARE-encoding Syx $1 A$. The enhanced wing-nicking phenotype induced by the dNSF2 $2 \mathrm{Q} / \mathrm{Q}$ construct we observed in various doc mutant backgrounds also suggests a sensitivity to events at the target membrane, given the role of NSF proteins in SNARE complex disassembly (Hanson \& Whiteheart 2005).

A notable feature of the Yip superfamily in other systems is that each has several different genes encoding YIP proteins, including four in yeast and nine in mammals (Shakoori et al. 2003). The Drosophila Yip superfamily matches that of yeast, with homologs of the Yip1p, Yip4p and Yip5p proteins encoded by the currently unstudied genes CG12404, CG3652 and CG4645 respectively. The expansion of the family to nine in mammals has functional significance, generating isoforms with distinct, tissue-specific roles. For example, Yif1B is involved in targeting of the serotonin receptor in dendrites, whereas Yif1A is involved in ERGolgi transport (Carrel et al. 2008). Mice homozygous for a null mutation in another family member, YIPF6, have recently been shown to have a specific defect in secretion from epithelial cells lining the intestine (Brandl et al. 2012). Alternative splicing of the doc mRNA produces three different Doc isoforms, differing at their N-terminal end. Although the differences between 
400 the Doc A, B and C subtypes are modest, we found that the splice donor and acceptor sites for 401 these alternative splice events are conserved in the doc homologs of many other Drosophila 402 species, suggesting that this same set of three Doc proteins are also produced in other members 403 of the genus and are therefore likely to represent functionally distinct isoforms. Furthermore, the 404 location of this variation is highly significant, because the hydrophilic N-terminal end of YIP 405 family members is predicted to project into the cytoplasm (Matern et al. 2000; Yang et al. 1998), 406 where it interacts with a variety of other proteins such as Rab-family GTPases that play crucial

407 408 409 410
Of the four $d o c$ alleles originally identified, and the three that we have examined in detail, $d o c^{1}$ is unique in both its viability in homozygous condition and in producing an oblique wing. Its status as a doc allele is confirmed by its rescue using the same transgene that restores viability to the two lethal alleles we tested, so what kind of allele does $d o c^{l}$ represent? Nonlethality and straightforward germline rescue strongly suggests a hypomorph, but none of the lethal $d o c$ alleles show any visible phenotype when heterozygous, and the semi-dominance of the $d o c^{1}$ oblique wing phenotype also seems at odds with this interpretation. It cannot be hypermorphic either, because increased wild-type dosage from the $d o c^{+}$genomic construct restores the wildtype wing, rather than making it worse. A third possibility is that $d o c^{l}$ is neomorphic, whereas the lethal alleles are nulls or hypomorphs. This model would be consistent with its semi-dominance and unique wing phenotype, but again this interpretation does not sit easily with straightforward recovery of the wild-type wing by the genomic rescue construct, as well as the qualitatively similar interactions of $d o c^{1}$ and $d o c^{16}$ or $d o c^{99}$ with $d N S F 2^{E / Q}$, miniature and Notch pathway components (Figures 3, $4 \& 5$ ). The ability of the genomic construct to rescue the $d o c^{l}$ oblique wing would suggest that the mutant defect is present within the region of the genome covered by our transgene. However, we have been unable to identify any molecular defects unique to the $d o c^{1}$ chromosome within this region, despite resequencing this genomic interval multiple times. We also could not identify a mutation in the $d o c^{99}$ chromosome, but in this case the lethality of the chromosome means there is always a need for a $\mathrm{doc}^{+}$chromosome present in the sequencing reaction; therefore the wild-type sequence may have hidden a mutant 
431 peak in the chromatogram. However, the $d o c^{l}$ mutation is viable and therefore can be sequenced 432 in homozygous condition, so there must be an alternative explanation for this allele. The most 433 likely explanation is that a mutation outside the sequenced region - in a distant enhancer for 434 example - that alters expression of an otherwise wild-type $d o c$, in a way that disturbs wing 435 development without affecting viability. However, for this model to explain the $d o c^{1}$ phenotype 436 the rescue construct would have to insert in a genomic location that provided the missing 437 expression in the wing. An alternative and possibly less parsimonious explanation is to invoke 438 epigenetics: perhaps a heritable change in chromatin structure is altering the pattern of $d o c$ expression and a wild-type copy of the gene in a different epigenetic landscape corrects the problem. This would be an unusual explanation, but such epigenetic modification does occurs in Drosophila, driven by Polycomb and Trithorax group proteins (Ruden \& Lu 2008). Sequencing of adjacent sequences beyond the region present in the transgene might reveal additional changes, but confirming that a change on the $d o c^{1}$ chromosome outside the transcribed region is actually causative would not be straightforward. It may be that isolation of new doc alleles with oblique wing phenotypes and an identifiable molecular lesion will be required to solve the conundrum of $d o c^{1}$.

\section{A broad role for Doc in vesicle trafficking}

We found that loss of $d o c$ in the developing wing margin leads to nicks very similar to those produced by some alterations of Notch pathway signaling. Over-expression of Delta was able to restore normal development of the wing blade, indicating that reduced Notch pathway activity was responsible for the marginal nicks; in addition, this result shows that ligand availability is the limiting factor, because Serrate over-expression also produced limited rescue, whereas increased Notch receptor expression did not produce any rescue. The Delta/Serrate rescue of the RNAi-induced nicks also demonstrates that vesicle trafficking pathways are not blocked altogether by expression of the $d o c$-RNAi construct, otherwise Delta overexpression would have no effect. This may reflect incomplete knockdown of $d o c^{+}$by the $d o c-$ RNAi construct, but could also be explained by the presence of a redundant, and Doc-independent, mechanism of Delta transport. It is surprising to us that the doc-knockdown-induced nicks could be rescued by increased availability of a single protein, Delta, given that we would expect ER- 
462 Golgi transport of a plethora of proteins to be significantly reduced. This might reflect a role for

463 Doc in some, but not all, COPII-mediated ER-Golgi vesicle transport, or perhaps might be due to

464 a very brief window of expression induced by the C96-GAL4 driver, during which Delta

465 transport is critical. However, our results do confirm that transport of other proteins also depends

466 on Doc, because although Delta overexpression permitted normal development of cells in the

467 wing blade, these otherwise-rescued doc-RNAi wings lack many marginal bristles (Fig. 4H).

468 Development of these bristles is known to depend on Wingless-mediated signaling (Zhang \&

469 Carthew 1998), so this strongly suggests that another critically important developmental ligand

470 relies on Doc-mediated vesicle transport. We found additional evidence that Doc also plays a key

471 role in earlier developmentally important signaling events, such as the finding that some

472 heterozygous combinations of $N$ and $d o c$ alleles generated synthetic lethality. These results are

473 consistent with a broad role for Doc in vesicle trafficking.

474

475 The link between doc and dumpy

We originally became interested in docked because $d o c^{1}$ homozygous flies show an

indentation in the distal tip of the wing that is indistinguishable from the phenotype observed in

478

479

480

481

482

483

484

485

486

487

488

489

490

491

492

the oblique class of dumpy alleles, raising the possibility that the two genes are functionally connected. We have been able to show here, in two distinct ways, that the similarity in phenotype indeed reflects a common mechanism. First of all, there is a strong non-additive interaction between $d o c^{1}$ and three different $d p y$ alleles, perhaps the most striking example being between $d p y^{o l v r}$ and $d o c^{1}$. Heterozygous animals for the former have wild-type wings, and $d o c^{l}$ heterozygotes have only a very slight truncation at the distal tip of the wing; by contrast, animals heterozygous for both $d o c^{l}$ and $d p y^{o l v r}$ show a strong oblique phenotype (Fig. 1). Second, having shown that Doc encodes a protein likely to be involved in ER-Golgi transport we found that reduced dosage of sarl and sec13, both of which encode elements of COPII vesicles, also produces a marked enhancement of the oblique wing phenotype of $d p y^{D}$. This unambiguously establishes that reduced COPII vesicle trafficking exacerbates dumpy oblique mutations, presumably by limiting transport of Dpy during wing development. Therefore, given that Doc also plays a role in vesicle trafficking, the simplest explanation of the dumpy-like wing truncation of $d o c^{1}$ is that it results from reduced availability of Dpy. This model has an additional implication: if an oblique wing phenotype can be produced by reducing the concentration of

Peer] reviewing PDF | (2018:10:31761:1:1:NEW 19 Aug 2021) 
493 wild-type Dpy in the wing, then the simplest explanation of the dumpy oblique alleles is that they

494 are hypomorphs. Also consistent with this model is the sensitivity we observed of Dpy to

495 reduced vesicle trafficking, for example in the much-increased oblique wing we observed in

$496 d p y^{D}$ when dosage of sarl or sec13 was halved (Fig. 5).

497 The precise lesion in a large collection of $d p y$ mutations has been determined,

498 revealing that most alleles are caused by truncating mutations: premature stop codons, deletions

499 or those affecting splice sites (Carmon et al. 2010). However, the majority of the non-lethal

500 oblique alleles show a different pattern, not only because the mutations are clustered in a single

501 exon, but also because they are missense. Based on patterns of sequence conservation described

502 in the same study, it was proposed that this exon is alternatively spliced. It may be then that $d p y^{o}$

503 mutations reduce the availability of a wing-specific isoform, resulting in the observed

504 combination of a homozygous viable, oblique wing phenotype. However, it remains something

505 of a mystery what role might normally be played by such a wing-specific Dumpy isoform, and

506 how a reduced amount of it generates reduced growth in such a specific location. The exon in

507 which the non-lethal oblique mutations occur contains several EGF repeats, so one possibility is

508 that these repeats are modified by Eogt in a way that is necessary for Dpy function during wing

509 morphogenesis.

510

511 A puzzling aspect of the doc-dpy interaction is why the enhanced dumpy oblique

512 phenotypes are only observed with $d o c^{1}$, and not the lethal alleles $d o c^{16}$ and $d o c^{99}$. If the

513 interaction between $d o c^{1}$ and dumpy is simply from restricted ER-Golgi transport of Dpy, we

514 would expect reduced dosage of the more severe alleles of doc to enhance the oblique wing

515 phenotype of $d p y$ alleles at least as much as $d o c^{l}$. Part of the answer to this puzzle, as discussed

516 above, probably rests on the unresolved qualitative difference between the $d o c^{1}$ and $d o c^{16 / 99}$

517 alleles. However, the range of $d o c$ allele phenotypes also echoes the behavior of the different

518 classes of $d p y$ alleles, which can produce three phenotypes (lethality, thoracic vortices, oblique

519 wings) alone, or in any combination with each other (Carlson 1959). It may be that this is an

520 example of an allele-specific interaction, perhaps due to a neomorphic change in the $d o c$

521 expression pattern in the developing wing in $d o c^{1}$ that is absent from the other $d o c$ alleles. 

machinery, it would be surprising if the interaction we observed between $d o c$ and $d p y$ is unique; unless Doc is only involved in trafficking a limited range of cargoes, interactions with other genes encoding secreted or membrane-bound proteins would be expected. The size of Dpy does not appear to be a critical determinant of the interaction, because we found no hint that mutation of several other genes encoding large cargoes results in a genetic interaction with doc. Dpy contains a ZP domain, so we looked for genetic interaction with ten different genes encoding ZP domain proteins; these all share a similar final destination, and may have related roles (Jovine et al. 2002) In this case we did find one gene with a clear interaction: miniature. Again, the interaction does not point toward size as being important, because Min is of an unremarkable size, only 682 amino acids long. The interaction extended to multiple alleles of both genes and was non-additive, producing markedly enhanced miniature phenotypes with just a halving of $d o c$ dosage, even with doc alleles that have no wing phenotype on their own. In combination with a small deficiency that uncovers miniature, $D f(1) m 259-4$, heterozygous combinations of two doc alleles with no phenotype alone generated a synthetic lethal when combined. Presumed loss of function miniature alleles are homozygous viable (Roch et al. 2003), so this lethality must depend on the combined effect of reduced $m$ dosage in the context of reduced transport of other molecules, resulting from the lowered availability of Doc. What then is the common feature underlying the strong $d o c-d p y$ and $d o c-m$ interactions? Dpy and Min are both components of the ECM, and it has become clear that, in addition to a variety of structural functions, the ECM is intimately involved in regulating the distribution, activation and delivery of a wide variety of hormones and morphogens (Hynes 2009). This seems to be the case for Min, because recent evidence suggests that a major role for Min in the wing is in regulating diffusion of the insect tanning hormone Bursicon through the ECM (Bilousov et al. 2012). It may be that reduced dosage of $m$, in combination with a doc-dependent reduction in traffic of both Min and other ECM components such as Dpy, disrupts Bursicon signaling enough to produce the enhanced miniature wing phenotypes.

Although the precise role of Yif homologs in vesicle transport remains uncertain, the

552 function of the Yip-Yif complex is highly conserved in eukaryotes. This was most dramatically demonstrated in yeast, by rescue of the lethal defect in Yipl mutants when transformed with a 
554 vector expressing human YIP1A (Chen \& Collins 2005). Functional conservation of this family 555 from fungi to mammals suggests very strongly that Doc is likely to play a similar role in 556 Drosophila. We have shown here that $d o c$ encodes the sole Yif homolog Drosophila and 557 anticipate that further study of docked may help to clarify the role Yif proteins in vesicle 558 trafficking in eukaryotes.

559

\section{Acknowledgements}

561

562 We thank T. Abbeyquaye for his contributions at the origin of this work; undergraduate members 563 of the Thackeray lab who participated in the project; the Bloomington Drosophila Stock Center, 564 the Vienna Drosophila Resource Center and M. Bender for stocks. Flybase was used in a wide 565 variety of ways and was critical to the success of this project. 
566

567

568

569

570

571

572

573

574

575

576

577

578

579

580

581

582

583

584

585

586

587

588

589

590

591

592

593

594

595

596

597

598

599

600

601

602

603

604

605

606

607

608

609

\section{References}

Artavanis-Tsakonas S, Rand MD, and Lake RJ. 1999. Notch signaling: cell fate control and signal integration in development. Science 284:770-776.

Baker NE. 2007. Patterning signals and proliferation in Drosophila imaginal discs. Curr Opin Genet Dev 17:287-293. 10.1016/j.gde.2007.05.005

Banerjee A, Barry VA, DasGupta BR, and Martin TF. 1996. N-Ethylmaleimide-sensitive factor acts at a prefusion ATP-dependent step in $\mathrm{Ca} 2+$-activated exocytosis. $J$ Biol Chem 271:20223-20226.

Barrowmann J, Wang W, Zhang Y, and Ferro-Novick S. 2003. The Yip1p.Yif1p complex is required for the fusion competence of endoplasmic reticulum-derived vesicles. $J$ Biol Chem 278:19878-19884. 10.1074/jbc.M302406200

Bilousov OO, Kozeretska IA, and Katanaev VL. 2012. Role of the gene Miniature in Drosophila wing maturation. Genesis 50:525-533. 10.1002/dvg.22016

Bokel C, Prokop A, and Brown NH. 2005. Papillote and Piopio: Drosophila ZP-domain proteins required for cell adhesion to the apical extracellular matrix and microtubule organization. Journal of cell science 118:633-642.

Brandl K, Tomisato W, Li X, Neppl C, Pirie E, Falk W, Xia Y, Moresco EM, Baccala R, Theofilopoulos AN, Schnabl B, and Beutler B. 2012. Yip1 domain family, member 6 (Yipf6) mutation induces spontaneous intestinal inflammation in mice. Proc Natl Acad Sci U S A 109:12650-12655. 10.1073/pnas.1210366109

Carlson EA. 1959. Allelism, Complementation, and Pseudoallelism at the Dumpy Locus in Drosophila Melanogaster. Genetics 44:347-373.

Carmon A, Guertin MJ, Grushko O, Marshall B, and MacIntyre R. 2010. A Molecular Analysis of Mutations at the Complex dumpy Locus in Drosophila melanogaster. PLoS One 5:e12319.

Carrel D, Masson J, Al Awabdh S, Capra CB, Lenkei Z, Hamon M, Emerit MB, and Darmon M. 2008. Targeting of the 5-HT1A serotonin receptor to neuronal dendrites is mediated by Yif1B. The Journal of Neuroscience 28:8063-8073.

Chen CZ, and Collins RN. 2005. Insights into biological functions across species: examining the role of Rab proteins in YIP1 family function. Biochem Soc Trans 33:614-618.

de Celis JF, Garcia-Bellido A, and Bray SJ. 1996. Activation and function of Notch at the dorsalventral boundary of the wing imaginal disc. Development 122:359-369.

Etournay R, Popovic M, Merkel M, Nandi A, Blasse C, Aigouy B, Brandl H, Myers G, Salbreux G, Julicher F, and Eaton S. 2015. Interplay of cell dynamics and epithelial tension during morphogenesis of the Drosophila pupal wing. Elife 4:e07090. 10.7554/eLife.07090

Gurkan C, Stagg SM, LaPointe P, and Balch WE. 2006. The COPII cage: unifying principles of vesicle coat assembly. Nature Reviews Molecular Cell Biology 7:727-738.

Gustafson K, and Boulianne GL. 1996. Distinct expression patterns detected within individual tissues by the GAL4 enhancer trap technique. Genome 39:174-182.

Hanson PI, and Whiteheart SW. 2005. AAA+ proteins: have engine, will work. Nat Rev Mol Cell Biol 6:519-529. 10.1038/nrm1684

Heidtman M, Chen CZ, Collins RN, and Barlowe C. 2003. A role for Yip1p in COPII vesicle biogenesis. J Cell Biol 163:57-69. 10.1083/jcb.200306118

Peer] reviewing PDF | (2018:10:31761:1:1:NEW 19 Aug 2021) 
610 Heidtman M, Chen CZ, Collins RN, and Barlowe C. 2005. Yos 1p is a novel subunit of the 611 Yiplp-Yiflp complex and is required for transport between the endoplasmic reticulum 612 and the Golgi complex. Mol Biol Cell 16:1673-1683. E04-10-0873

613 Hynes RO. 2009. The extracellular matrix: not just pretty fibrils. Science 326:1216-1219.

$614 \quad 10.1126 /$ science. 1176009

615

616

617

618

619

620

621

622

623

624

625

626

627

628

629

630

631

632

633

634

635

636

637

638

639

640

641

642

643

644

645

646

647

648

649

650

651

652

653

654

655

Jin C, Zhang Y, Zhu H, Ahmed K, Fu C, and Yao X. 2005. Human Yip1A specifies the localization of Yifl to the Golgi apparatus. Biochemical and biophysical research communications 334:16-22.

Jovine L, Qi H, Williams Z, Litscher E, and Wassarman PM. 2002. The ZP domain is a conserved module for polymerization of extracellular proteins. Nat Cell Biol 4:457-461. $10.1038 / \mathrm{ncb} 802$

Lee S, and Cooley L. 2007. Jagunal is required for reorganizing the endoplasmic reticulum during Drosophila oogenesis. The Journal of cell biology 176:941-952.

Lord C, Ferro-Novick S, and Miller EA. 2013. The highly conserved COPII coat complex sorts cargo from the endoplasmic reticulum and targets it to the golgi. Cold Spring Harb Perspect Biol 5. 10.1101/cshperspect.a013367

Malhotra V, and Erlmann P. 2011. Protein export at the ER: loading big collagens into COPII carriers. EMBO J 30:3475-3480. 10.1038/emboj.2011.255

Matern H, Yang X, Andrulis E, Sternglanz R, Trepte HH, and Gallwitz D. 2000. A novel Golgi membrane protein is part of a GTPase-binding protein complex involved in vesicle targeting. The EMBO Journal 19:4485-4492.

Matsuoka K, Orci L, Amherdt M, Bednarek SY, Hamamoto S, Schekman R, and Yeung T. 1998. COPII-coated vesicle formation reconstituted with purified coat proteins and chemically defined liposomes. Cell 93:263-275. S0092-8674(00)81577-9 [pii]

Matusek T, Djiane A, Jankovics F, Brunner D, Mlodzik M, and Mihaly J. 2006. The Drosophila formin DAAM regulates the tracheal cuticle pattern through organizing the actin cytoskeleton. Development 133:957-966. 10.1242/dev.02266

McQuilton P, Pierre SES, and Thurmond J. 2011. FlyBase 101 - the basics of navigating FlyBase. Nucleic Acids Research 40:D706-D714.

Neto-Silva RM, Wells BS, and Johnston LA. 2009. Mechanisms of growth and homeostasis in the Drosophila wing. Annu Rev Cell Dev Biol 25:197-220. 10.1146/annurev.cellbio.24.110707.175242

Neumann CJ, and Cohen SM. 1996. A hierarchy of cross-regulation involving Notch, wingless, vestigial and cut organizes the dorsal/ventral axis of the Drosophila wing. Development 122:3477-3485.

Panin VM, and Irvine KD. 1998. Modulators of Notch signaling. Semin Cell Dev Biol 9:609-617. S1084-9521(98)90263-4

Prout M, Damania Z, Soong J, Fristrom D, and Fristrom JW. 1997. Autosomal mutations affecting adhesion between wing surfaces in Drosophila melanogaster. Genetics 146:275285.

Rayburn LY, Gooding HC, Choksi SP, Maloney D, Kidd AR, 3rd, Siekhaus DE, and Bender M. 2003. amontillado, the Drosophila homolog of the prohormone processing protease PC2, is required during embryogenesis and early larval development. Genetics 163:227-237.

Reichman-Fried M, and Shilo BZ. 1995. Breathless, a Drosophila FGF receptor homolog, is required for the onset of tracheal cell migration and tracheole formation. Mech Dev $52: 265-273$. 
656

657

658

659

660

661

662

663

664

665

666

667

668

669

670

671

672

673

674

675

676

677

678

679

680

681

682

683

684

685

686

687

688

689

690

691

692

693

694

695

696

697

698

699

700

Rios-Barrera LD, Sigurbjornsdottir S, Baer M, and Leptin M. 2017. Dual function for Tango1 in secretion of bulky cargo and in ER-Golgi morphology. Proc Natl Acad Sci U S A 114:E10389-E10398. 10.1073/pnas.1711408114

Roch F, Alonso CR, and Akam M. 2003. Drosophila miniature and dusky encode ZP proteins required for cytoskeletal reorganisation during wing morphogenesis. J Cell Sci 116:11991207.

Ruden DM, and Lu X. 2008. Hsp90 affecting chromatin remodeling might explain transgenerational epigenetic inheritance in Drosophila. Curr Genomics 9:500-508. $10.2174 / 138920208786241207$

Sakaidani Y, Nomura T, Matsuura A, Ito M, Suzuki E, Murakami K, Nadano D, Matsuda T, Furukawa K, and Okajima T. 2011. O-linked-N-acetylglucosamine on extracellular protein domains mediates epithelial cell-matrix interactions. Nat Commun 2:583. 10.1038/ncomms 1591

Shakoori A, Fujii G, Yoshimura S, Kitamura M, Nakayama K, Ito T, Ohno H, and Nakamura N. 2003. Identification of a five-pass transmembrane protein family localizing in the Golgi apparatus and the ER. Biochem Biophys Res Commun 312:850-857. 10.1016/j.bbrc.2003.10.197

Slatis HM, and Willermet DA. 1954. The Miniature Complex in Drosophila Melanogaster. Genetics 39:45-58.

St Pierre SE, Ponting L, Stefancsik R, and McQuilton P. 2014. FlyBase 102--advanced approaches to interrogating FlyBase. Nucleic Acids Res 42:D780-788. 10.1093/nar/gkt1092

Stewart BA, Mohtashami M, Zhou L, Trimble WS, and Boulianne GL. 2001. SNARE-dependent signaling at the Drosophila wing margin. Dev Biol 234:13-23. 10.1006/dbio.2001.0228

Syed ZA, Hard T, Uv A, and van Dijk-Hard IF. 2008. A potential role for Drosophila mucins in development and physiology. PLoS One 3:e3041. 10.1371/journal.pone.0003041

Thomas U, Speicher SA, and Knust E. 1991. The Drosophila gene Serrate encodes an EGF-like transmembrane protein with a complex expression pattern in embryos and wing discs. Development 111:749-761.

van de Hoef DL, Bonner JM, and Boulianne GL. 2013. FKBP14 is an essential gene that regulates Presenilin protein levels and Notch signaling in Drosophila. Development 140:810-819.

Venditti R, Wilson C, and De Matteis MA. 2014. Exiting the ER: what we know and what we don't. Trends Cell Biol 24:9-18. 10.1016/j.tcb.2013.08.005

Waddington C. 1940. The genetic control of wing development in Drosophila. Journal of Genetics 41:75-113. 10.1007/bf02982977

Wang, Q., Wang, Y., Yu, F., 2018 Yif1 associates with Yip1 on Golgi and regulates dendrite pruning in sensory neurons during Drosophila metamorphosis. Development 145: dev164475 doi: 10.1242/dev.164475

Wilkin MB, Becker MN, Mulvey D, Phan I, Chao A, Cooper K, Chung HJ, Campbell ID, Baron M, and MacIntyre R. 2000. Drosophila dumpy is a gigantic extracellular protein required to maintain tension at epidermal-cuticle attachment sites. Curr Biol 10:559-567.

Yang X, Matern HT, and Gallwitz D. 1998. Specific binding to a novel and essential Golgi membrane protein (Yip1p) functionally links the transport GTPases Ypt1p and Ypt31p. EMBO J 17:4954-4963. 10.1093/emboj/17.17.4954

PeerJ reviewing PDF | (2018:10:31761:1:1:NEW 19 Aug 2021) 
701 Zhang J, and Carthew RW. 1998. Interactions between Wingless and DFz2 during Drosophila $702 \quad$ wing development. Development 125:3075-3085.

703

704 


\section{Figure 1}

Allelic nature of doc mutations and interaction of $d o c^{1}$ with dumpy oblique alleles.

(A) Wildtype wing. (B-C) Wings of $d o c^{1}$ and $d p y^{\text {ov1 }}$ showing the oblique wing phenotype. (D)

Slight oblique wing phenotype in $\operatorname{doc}^{1}$ heterozygote. (E-F) Transheterozygous combinations of $d o c^{1}$ with $d o c^{16}$ and $d o c^{99}$ show the allelic nature of the three mutations. (G-L) Wings of $d o c^{1}$ with three different dumpy alleles show a strong interaction between the two genes. Representative wings from adults of the genotypes indicated; scale bar represents 100um. (M) Wing lengths of genotypes shown in panels G-L; error bars indicate standard error of the mean ( $n=25$ for $\mathrm{G}-$ J, $n=22$ for $K, n=17$ for $L$ ). Length was measured as a straight line from the intersection of the anterior crossvein with vein $L 3$, to the intersection of $L 3$ with the margin, using ImageJ. Asterisk indicates significant differences in wing lengths between $\mathrm{doc}^{+}$and $d^{1} c^{1} / d o c^{+}$siblings in each of the three dpy backgrounds; two-sample $t$ test assuming unequal variances, $d p y^{D}(d f=67) t=27.35, p=2.2 \times 10^{-38} ; d p y^{\text {DvlG5 }}(d f=89) t=19.55, p=2.87 \times 10^{-34} ; d p^{\text {olvr }}$ $(d f=21) t=10.66, p=3.13 \times 10^{-10}$. 


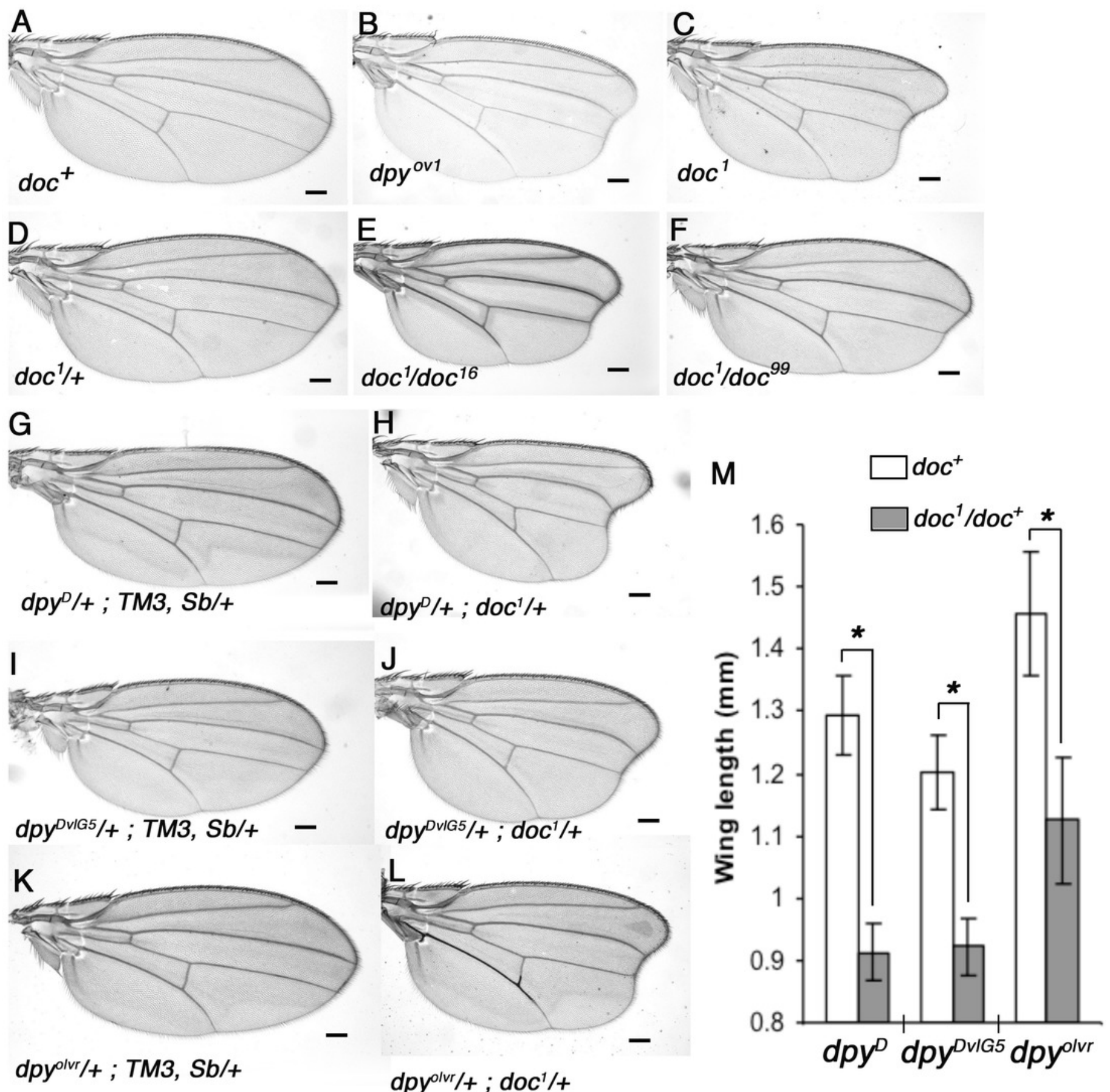




\section{Figure 2}

The doc complementation group corresponds to the gene model CG5484.

(A) Genomic DNA sequencing chromatograms of $d o c^{16}$ heterozygotes, showing that the same transition mutation is present (as a double peak) on both strands, producing a Q162STOP nonsense mutation. The affected glutamine codon is underlined in the upper chromatogram. B) The genomic region around CG5484; the $4.1 \mathrm{~kb}$ of DNA represented in the germline rescue construct is indicated by the red-shaded rectangle beneath the map. (C) Representative wing from $w^{1118}$; doc $c^{1}$ homozygote. (D) Representative wing from $w^{1118}$; doc $c^{1}$ homozygote carrying one copy of the $P\{w+, C G 5484\}$ construct shown in panel $B$; the oblique wing phenotype seen in panel $C$ is completely rescued in panel $D$. The animals in panels $C$ and $D$ are siblings; scale bars represent 100um. 
A $\operatorname{doc}^{+} \ldots$ MetGluAsnglnPheGlu...

doc $c^{16}$. MetGluAsn*

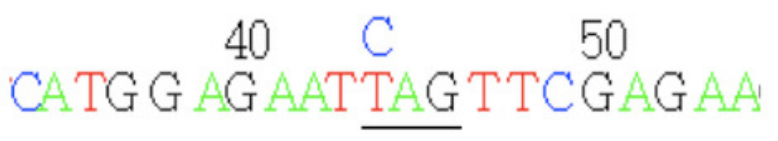

C
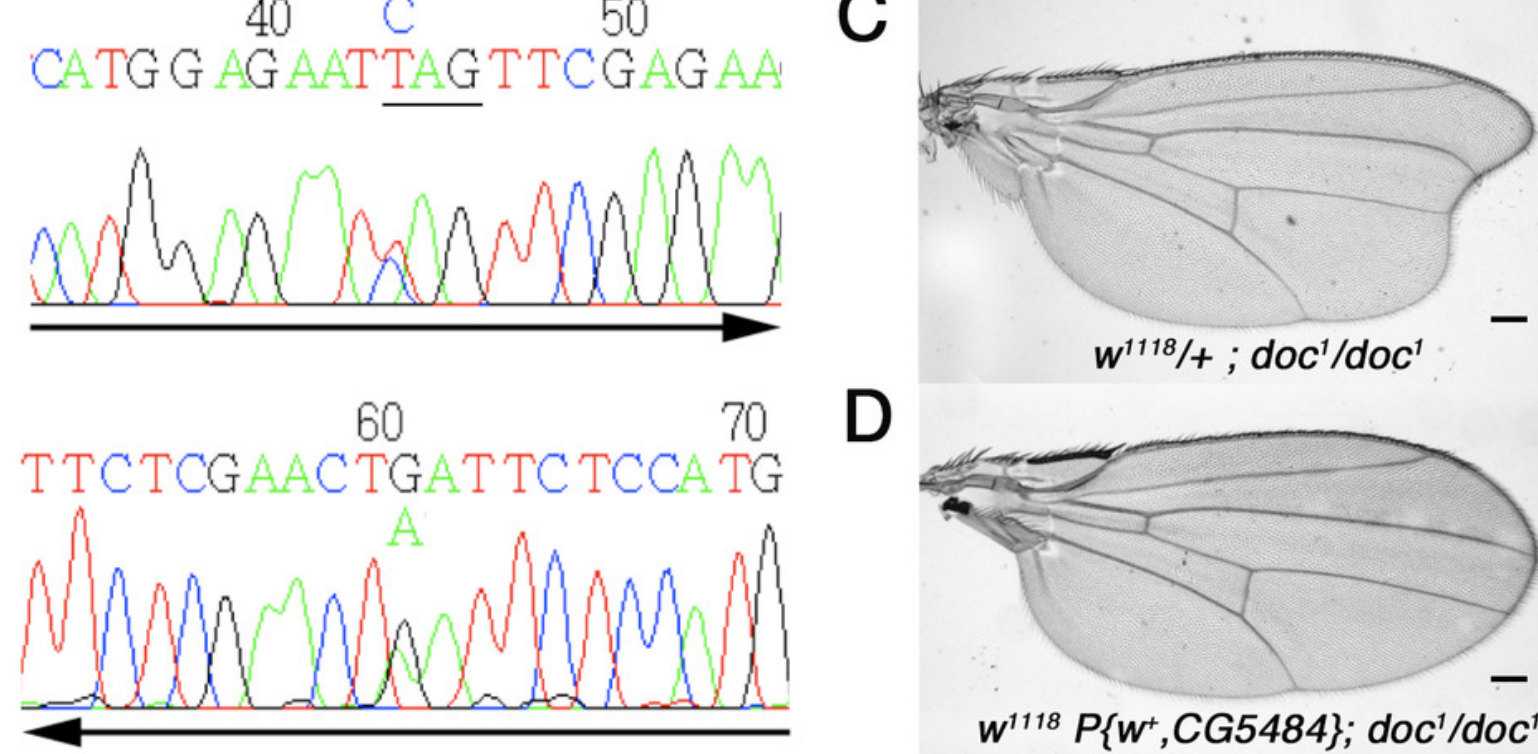

D

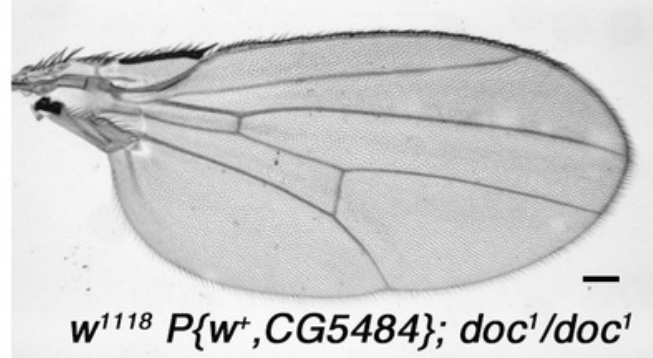

B

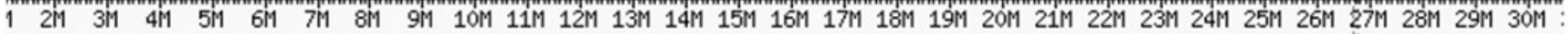

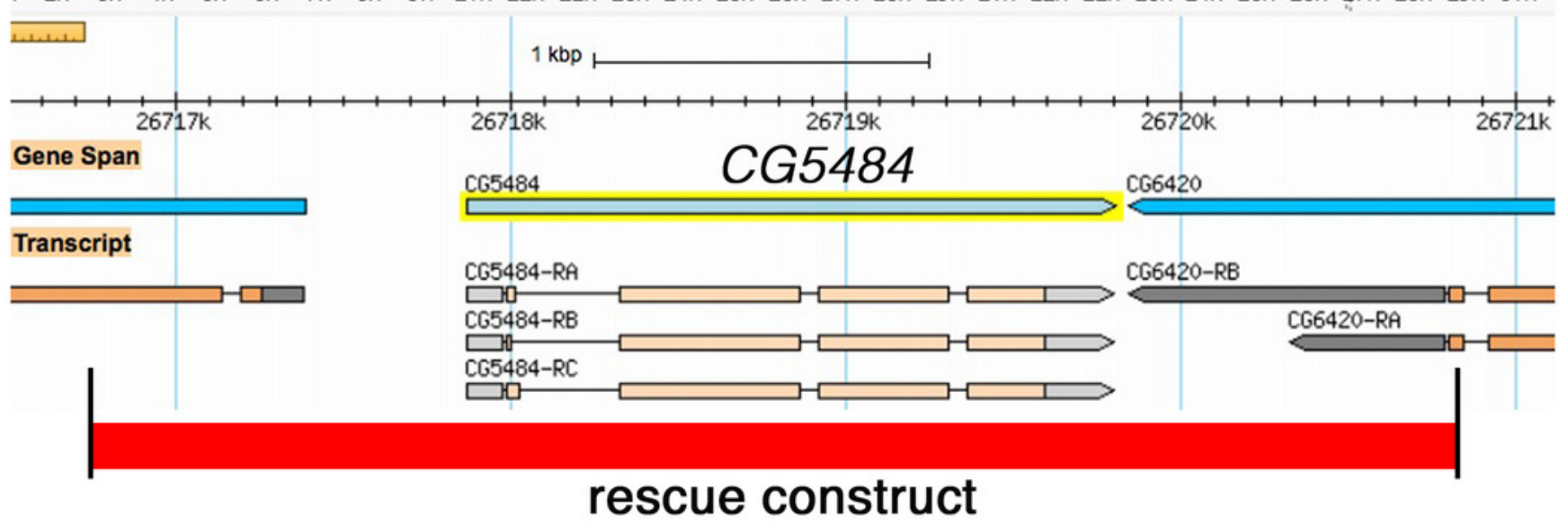




\section{Figure 3}

The doc alleles interact strongly with genes involved in vesicle trafficking.

(A-F) Strong enhancement of the oblique wing phenotype in $d^{1} c^{1}$ heterozygotes when also heterozygous for mutations in $\sec 23$ and $\sec 13$. (G-I) Enhanced wing phenotype of $d_{0 c}^{1}$ in a Syx1A heterozygous background. (J-L) Wing nicks in UAS-dNSF2 ${ }^{E / Q}$;C96-GAL4 wings are enhanced in backgrounds that are also heterozygous for $\operatorname{doc}^{1}$ and $d o c^{99}$. The same result was found for $\mathrm{doc}^{16}$ (not shown). Representative wings from adults of the genotypes indicated; scale bars represent 100um. 


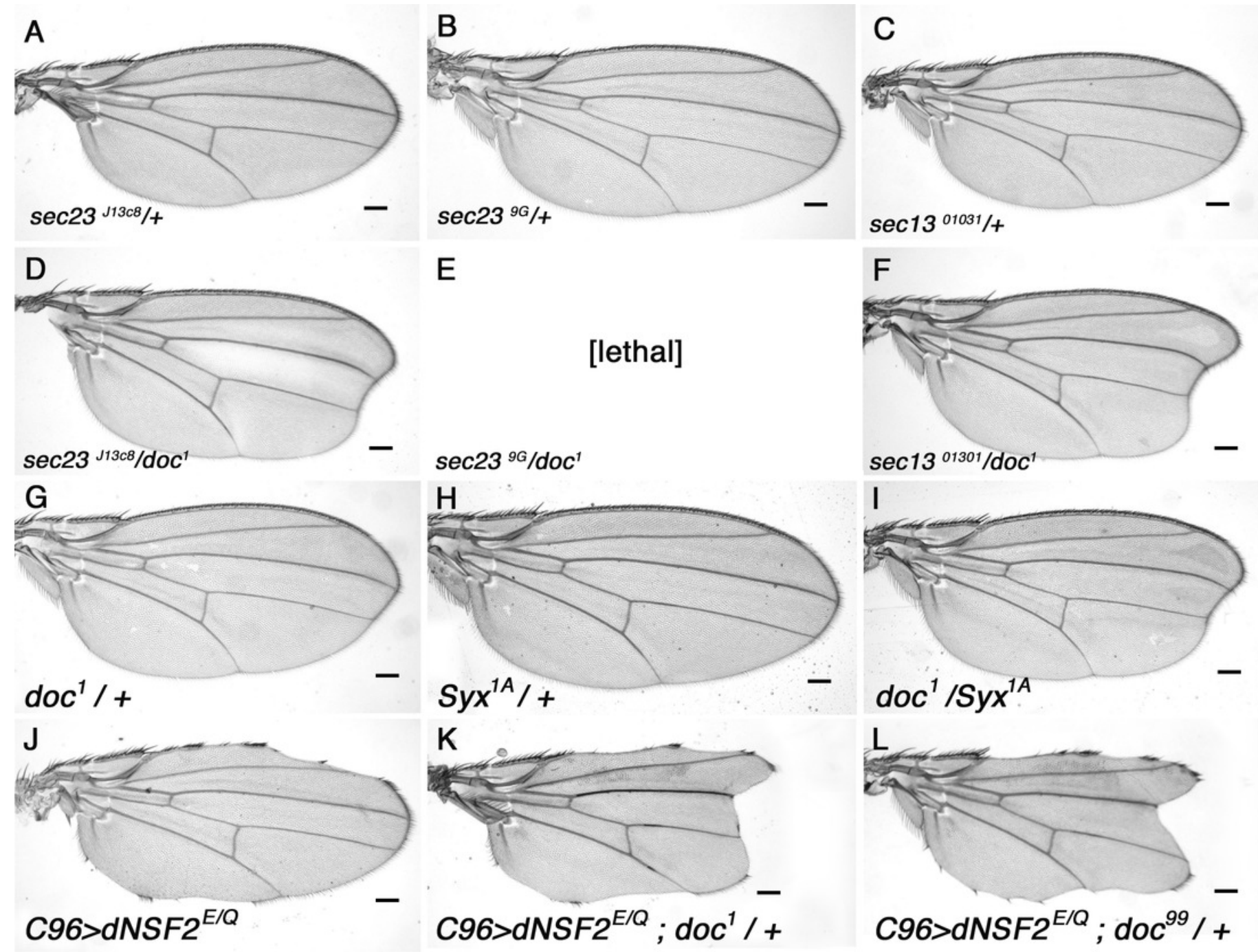


Figure 4

Genetic interaction of doc alleles with members of the Notch pathway.

(A-F) Mild enhancement of the Delta wing venation phenotype in $d o c^{1}$ and $d o c^{16}$ heterozygous backgrounds. (G-J) Wing nicks of UAS-Dicer2/+;UAS-RNAi-doc/+;C96-GAL4/+ are rescued by over-expression of Delta and Serrate, but not Notch. Representative wings from adults of the genotypes indicated; scale bars represent 100um.
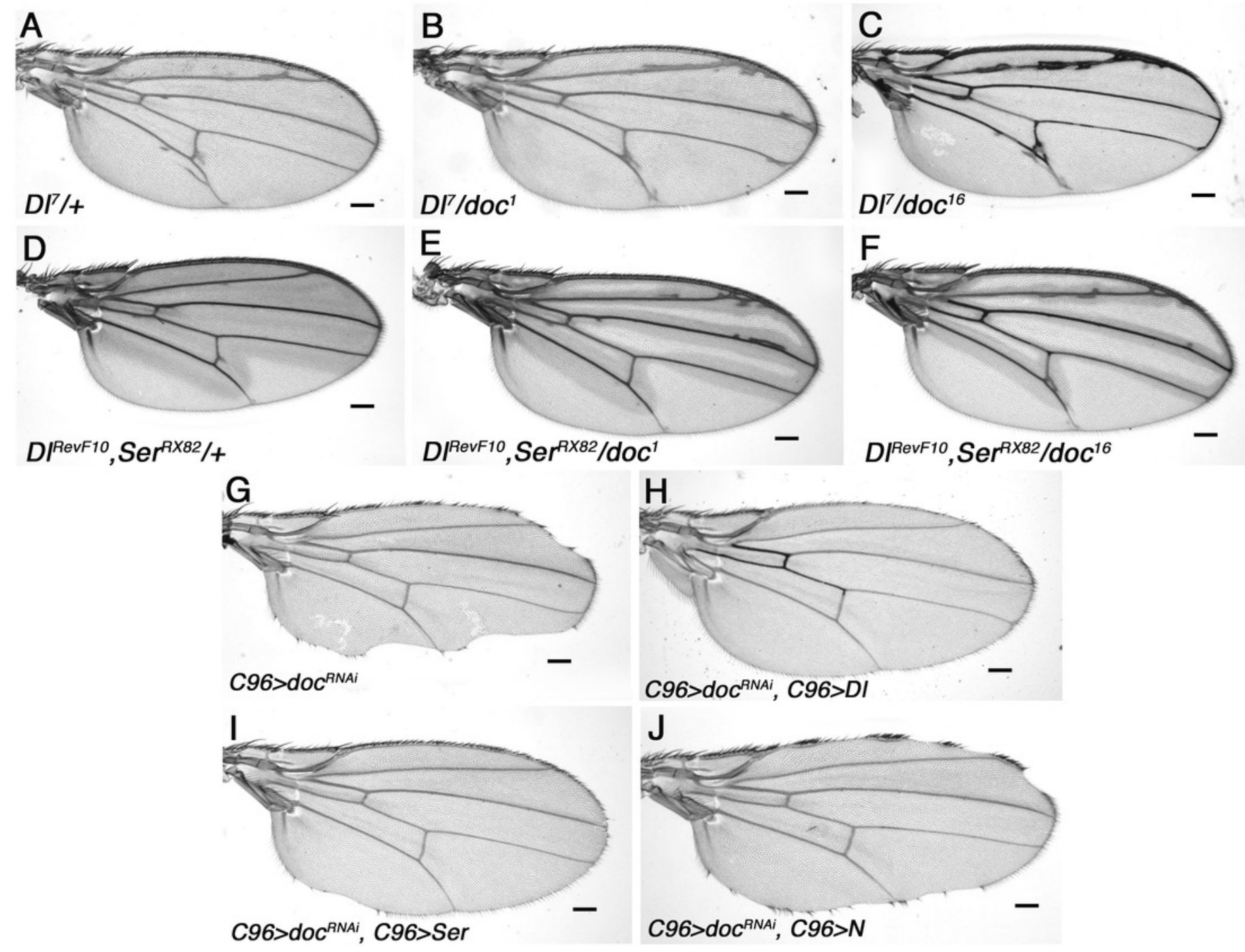


\section{Figure 5}

Vesicle trafficking mutations strongly enhance a dumpy dominant oblique mutation.

(A) Wings of the dominant oblique wing mutant $d p y^{D}$ show a near-wildtype wing. $(B, C)$ Wings of heterozygotes for either sar $1^{05712}$ or $\sec 13^{01031}$ are wild-type. (D,E) Animals simultaneously heterozygous for $d p y^{D}$ and either sar1 $1^{05712}$ or sec1 $3^{01031}$ have a strongly enhanced oblique wing phenotype. (F) Wing from a male $m^{1}$ hemizygote. (G-I) In backgrounds heterozygous for either $d o c^{1}$ or $d o c^{16}, m^{1}$ wings are crumpled, (I) whereas in $d o c^{99}$ heterozygotes the wings show excessive pigmentation. Representative wings from adults of the genotypes indicated; scale bars represent 100um.
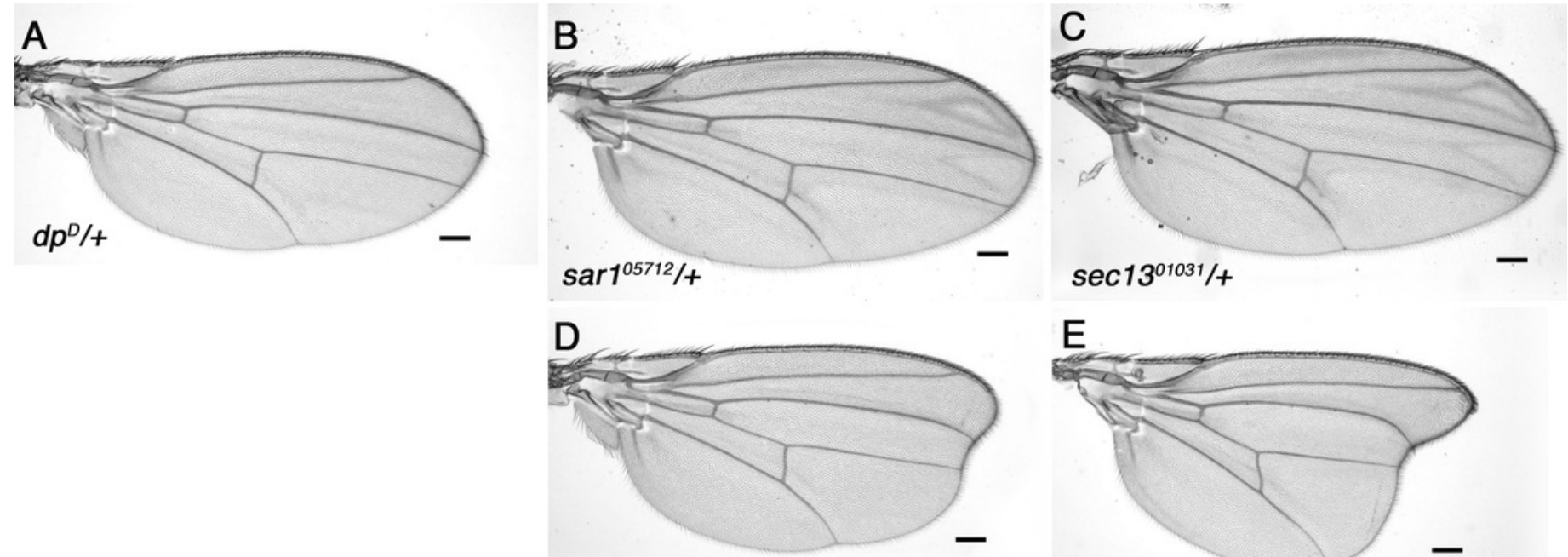

$d p^{D} /+; \operatorname{sar}^{105712 /+}$
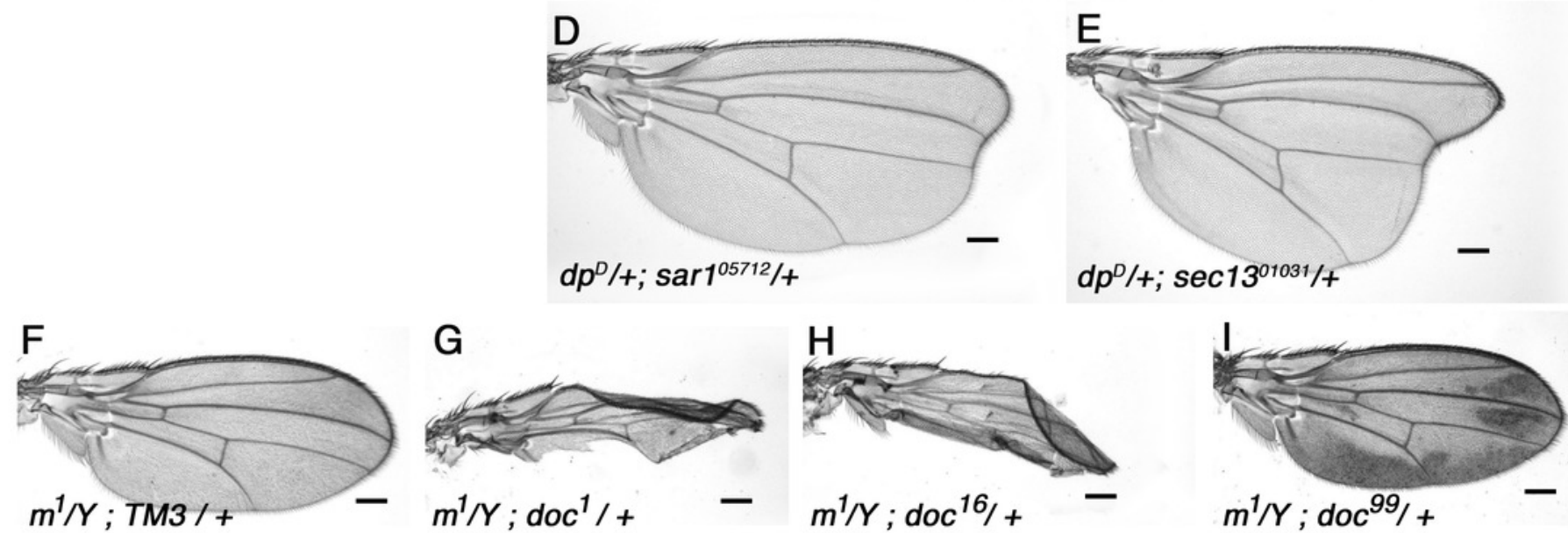